\title{
A Case Report of Impacted Fecalith Within Mucosal Pouch: An Unusual Cause of Colocolic Intussusception
}

\author{
Douglas Greer, Adrian Fernandez \\ Department of Surgery, South East Regional Hospital, Bega, NSW, Australia
}

Intussusception involving the colon is unusual in adults and when present is managed with resection due to the risk of malignancy. We present an unusual case where the intussusceptum was impacted stool in a mucosal pouch in the transverse colon. The patient presented with bleeding per rectum and abdominal pain and was found to have a colocolic intussusception on computed tomography. Colonoscopy showed an ulcerated mass in the transverse colon. A laparoscopic right hemicolectomy was performed. Histopathology demonstrated known chronic lymphocytic leukemia, but not solid malignancy. A large fecalith impacted within a mucosal pouch had acted as the lead point. This represents a highly unusual but benign cause of intussusception.

Keywords: Intussusception; Fecal impaction; Colectomy

\section{INTRODUCTION}

Intussusception is the process whereby a proximal segment of bowel (the intussusceptum) slides into a distal segment (intussuscipiens), often described as telescoping. It can be classified by location as enteroenteric, ileocolic, or colocolic. Although common and usually benign in children, intussusception is uncommon in adult patients and is most often caused by a pathological lead point, which is malignant in around $50 \%$ of cases $[1,2]$. In the colon, malignant lead points are largely adenocarcinoma, while benign lead points have a range of causes including polyps, lipomata, and lymphoid hyperplasia [2-4]. Fecalith within the appendix and cecum and bezoars within the terminal ileum have been described as lead points in ileocolic or colocolic intussusception $[5,6]$. We present a rare case of a fecalith in the transverse colon mimicking a submucosal lesion causing colocolic intussusception.

Received: Aug 10, 2021 - Revised: Sep 23, 2021 - Accepted: Sep 23, 2021 Correspondence to: Douglas Greer, B.Med, M. Clin Epi

Department of Surgery, South East Regional Hospital, 4 Virginia Drive, Bega, NSW 2550, Australia

Tel: +61-2-6491-9999, Fax: +61-2-8330-5525

E-mail: douglas.greer@health.nsw.gov.au

ORCID: https://orcid.org/0000-0001-6403-1381

(C) 2021 The Korean Society of Coloproctology

This is an open-access article distributed under the terms of the Creative Commons Attribution NonCommercial License (https://creativecommons.org/licenses/by-nc/4.0) which permits unrestricted noncommercial use, distribution, and reproduction in any medium, provided the original work is properly cited.

\section{CASE REPORT}

An 81-year-old male presented with bleeding per rectum for 1 day and intermittent abdominal pain for 2 weeks. He had no previous abdominal surgery and had never undergone a colonoscopy. He had a background of chronic lymphocytic leukemia (CLL) and was in remission having completed chemotherapy 1 year prior. He had normal vital signs and a soft, non-tender abdomen without palpable masses. The remainder of his physical examination was unremarkable. Laboratory investigations showed normal leukocytes, mild normocytic, hypochromic anemia (hemoglobin, $125 \mathrm{~g} / \mathrm{L}$; hematocrit, $0.37 \mathrm{~L} / \mathrm{L}$; mean corpuscular volume, $85 \mathrm{fL})$, and a mildly elevated C-reactive protein $(8 \mathrm{mg} / \mathrm{L})$. An abdominal computed tomography (CT) scan showed a colocolic intussusception in the right upper quadrant (Fig. 1).

A colonoscopy was performed preoperatively under sedation. A lesion was encountered in the transverse colon and the site was tattooed. The apex of the lesion showed mucosal ulceration (Fig. 2) although the appearance was not typical of malignancy. Navigation beyond the lesion was not possible due to luminal narrowing. The remainder of the colon distal to the lesion was unremarkable aside from sigmoid diverticulosis. We proceeded to perform a laparoscopic right hemicolectomy. At surgery, there was no clear intussusception as seen on the CT scan which was thought to reflect at least a partial reduction by colonoscopy. A firm mass was felt in the proximal transverse colon. An incidental Meckel's diverticulum was included in the resection and a stapled 


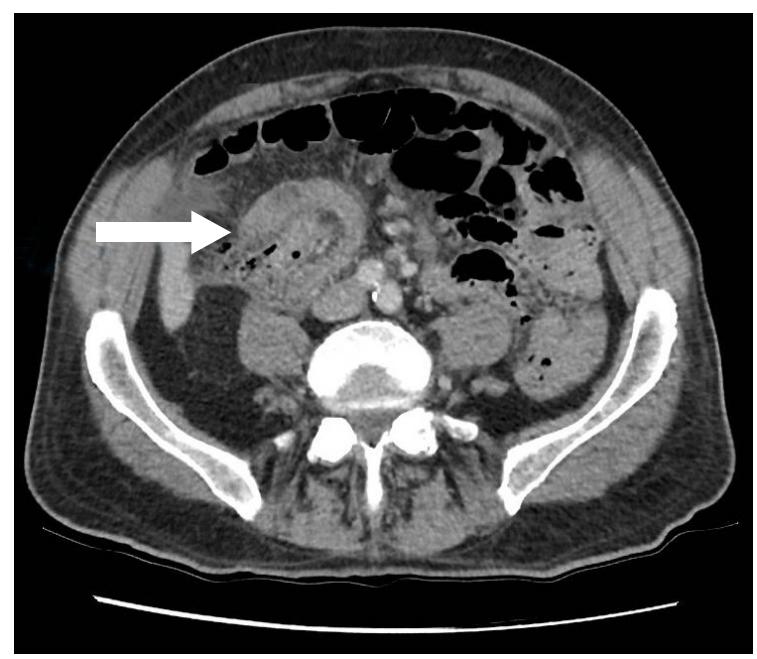

Fig. 1. Axial slice of computed tomography showing intussusception (arrow).

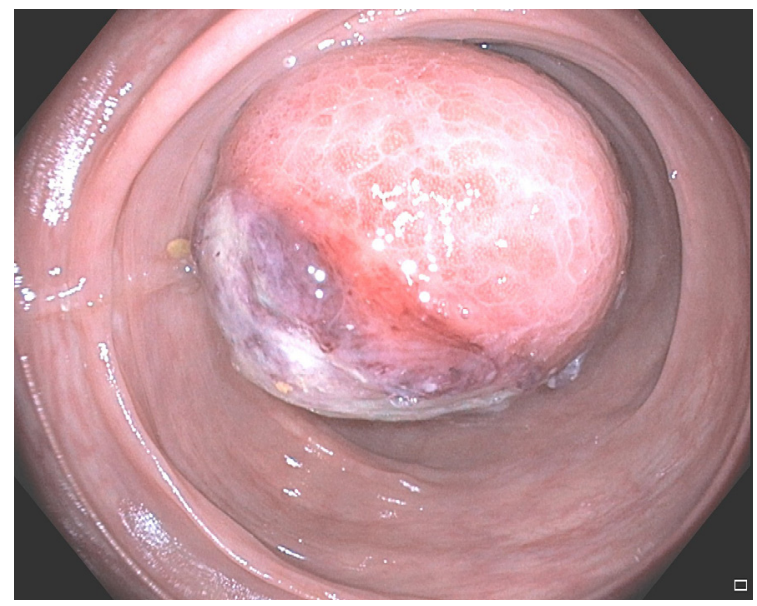

Fig. 2. Endoscopic photo showing intussusceptum with mucosal ulceration.

anastomosis formed.

Macroscopic examination of the resected specimen revealed a dome-shaped mass measuring $50 \times 40 \times 30 \mathrm{~mm}$ with surface ulceration within the luminal aspect of the proximal transverse colon. An apparent mucosa-lined pouch projecting into the lumen and filled with fecal material was seen on sectioning through the mass (Fig. 3). Microscopy reported a cyst-like space communicating with the lumen. The space was lined by mucosa and muscularis mucosa that invaginated into the submucosal layer and contained a large fecalith. There was extensive ulceration at the apex of the pouch; however, the remaining mucosa was unremarkable. Lymph nodes in the specimen were consistent with CLL. A 5-mm hyperplastic polyp was seen proximal to the pouch and the remainder of the specimen was unremarkable.

The patient's postoperative course was complicated by pneumo-

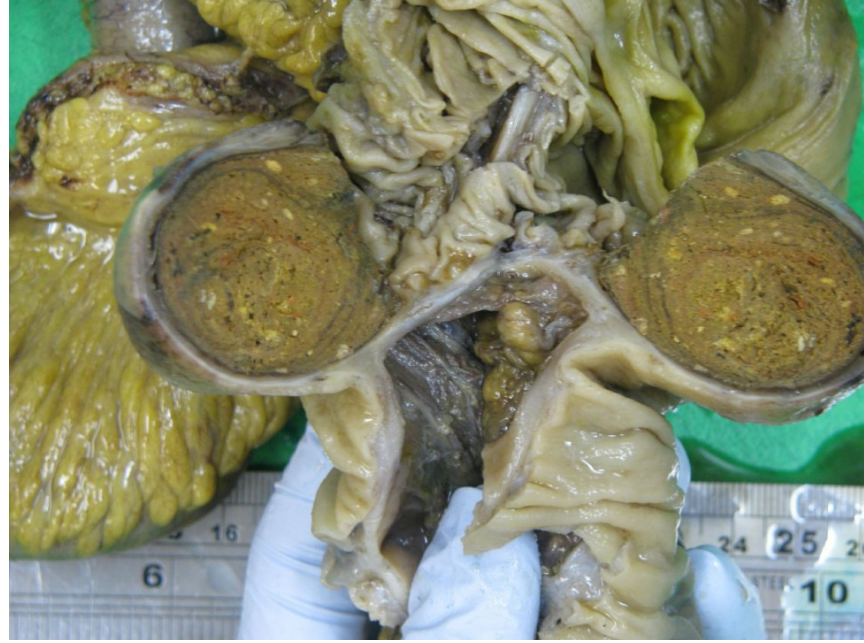

Fig. 3. Resected specimen sectioned through the mucosal pouch and contained fecalith.

nia which was managed with intravenous antibiotics, and ileus which resolved with conservative management. At follow-up, he had returned to baseline function and his bowels were working normally. He also had a scheduled appointment to meet with his hematologist to discuss the lymph nodal disease in the resected specimen reporting CLL.

Our Institutional Review Board does not review case reports according to institutional policy; informed consent was obtained from the patient for publication of this case report and accompanying images.

\section{DISCUSSION}

Intussusception remains uncommon in adults, it is estimated that it accounts for between $1 \%$ and $5 \%$ of bowel obstructions [3, 4]. Intussusceptions in adults are thought to involve the small bowel the majority of the time and where involving the large bowel alone are much more likely to represent a malignant process with estimates ranging from $50 \%$ to $100 \%$ in some series [2-4]. Fecalith within the appendix or ileocecal valve causing intussusception have been described in the literature [5, 7-9], and Adewale et al. [10] described a case of an intraluminal colonic fecalith causing intussusception in a cystic fibrosis patient. Our case differs significantly from this case due to the absence of predisposing factors for inspissated or impacted stool and the location of the fecalith.

It is not clear in this case whether a fecalith formed in a preexisting mucosal pouch, or whether an already formed fecalith 'burrowed' into the mucosa causing an invagination. Such a mucosal pouch may be caused by a giant colonic diverticulum (GCD), a rare presentation of diverticular disease which has been described as a cause of intussusception [11]. However, despite the patient having sigmoid diverticular disease, no right-sided diverticula were seen. This would make an isolated ascending colon GCD 
unusual [12].

Clinical presentations of adult intussusception also vary greatly. While some undoubtedly present with features of acute intestinal obstruction, this may only account for half [2]. In the remainder, the presentation is more insidious, with pain, nausea and bleeding variably seen, and a palpable mass infrequent $[2,4]$. These nonspecific features are encountered in colocolic intussusception, as in our case, and require a high index of suspicion to diagnose [3].

Due to the high incidence of malignancy, surgical resection is the mainstay of treatment of adult intussusception; however, the extent of resection has been controversial [2-4]. Formal oncological resection has been recommended especially in those over 60 years of age [4]. Laparoscopic or open reduction with limited resection has been described where a benign lead point is clearly identified [13]. Endoscopic treatment of an appendiceal fecalith causing intussusception was described by Lee et al. [7] but is not routinely performed.

Intussusception in adults is rare and may present a diagnostic dilemma. Although in this case we present an unusual benign cause, a lead point is common and is frequently malignant. Due to the incidence of a pathological lead point, resection is usually recommended.

\section{CONFLICT OF INTEREST}

No potential conflict of interest relevant to this article was reported.

\section{ACKNOWLEDGMENTS}

The authors appreciate Dr. Duncan McLeod, a pathologist of Westmead Hospital in Westmead, NSW, Australia for his valuable help.

\section{REFERENCES}

1. Azar T, Berger DL. Adult intussusception. Ann Surg 1997;226: 134-8.

2. Zubaidi A, Al-Saif F, Silverman R. Adult intussusception: a retrospective review. Dis Colon Rectum 2006;49:1546-51.

3. Coleman MJ, Hugh TB, May RE, Jensen MJ. Intussusception in the adult. Aust N Z J Surg 1981;51:179-80.

4. Marinis A, Yiallourou A, Samanides L, Dafnios N, Anastasopoulos G, Vassiliou I, et al. Intussusception of the bowel in adults: a review. World J Gastroenterol 2009;15:407-11.

5. Mahajna A, Krausz MM. Ileocolic intussusception in an adult patient due to a cecal fecalith. Isr Med Assoc J 2009;11:58-9.

6. Calero P, Scortechini M, Valiente J. A rare cause of intussusception in the adult: intestinal bezoar. Rev Gastroenterol Mex 2014; 79:145-6.

7. Lee CK, Lee SH, Park JY, Lee TH, Chung IK, Park SH, et al. Appendiceal intussusception due to a fecalith mimicking a submucosal tumor. Endoscopy 2009;41 Suppl 2:E25-6.

8. Meguro Y, Koide A, Tabuchi T. Submucosal tumor-like appendiceal intussusception as a result of fecalith. Dig Endosc 2014;26: 603.

9. Zhao H, Li Y, Cao D. Submucosal fecalith in the ileocecal valve. Gastrointest Endosc 2014;80:1183-4.

10. Adewale AT, Rowe SM, Solomon GM. Colocolonic intussusception in an adult cystic fibrosis patient. J Cyst Fibros 2019;18:e113.

11. Kim HJ, Kim JH, Moon OI, Kim KJ. Giant ascending colonic diverticulum presenting with intussusception. Ann Coloproctol 2013;29:209-12.

12. Nigri G, Petrucciani N, Giannini G, Aurello P, Magistri P, Gasparrini $\mathrm{M}$, et al. Giant colonic diverticulum: clinical presentation, diagnosis and treatment: systematic review of 166 cases. World J Gastroenterol 2015;21:360-8.

13. Wang LT, Wu CC, Yu JC, Hsiao CW, Hsu CC, Jao SW. Clinical entity and treatment strategies for adult intussusceptions: 20 years' experience. Dis Colon Rectum 2007;50:1941-9. 